\title{
DETERMINACIÓN EXPERIMENTAL DE LOS PARÁMETROS ÓPTIMOS DE OPERACIÓN EN EL PROCESO DE ELECTROLIXIVIACIÓN Y ELECTRODEPOSICIÓN SECUENCIAL DE ORO EN SOLUCIONES ÁCIDAS DE TIOUREA A PARTIR DE SULFUROS CONCENTRADOS
}

\author{
EXPERIMENTAL DETERMINATION OF THE OPTIMUM \\ OPERATING PARAMETERS ELECTRO LEACHING IN THE \\ PROCESS OF GOLD AND SEQUENTIAL \\ ELECTRODEPOSITION IN ACIDIC SOLUTIONS OF \\ THIOUREA FROM SULPHIDES CONCENTRATES
}

\author{
R.W Torres de la Cruz ${ }^{1}$ J. José Mejía ${ }^{2}$, W. Reátegui $\mathbf{R}^{3}$.
}

RESUMEN

\begin{abstract}
Se investigó a nivel de laboratorio las variables de operación (concentración de reactivos, pH y potencial de oxidación) del proceso de lixiviación de piritas auríferas procedentes de río Tablachaca (Ancash Perú) con Tiourea (TU) seguido de electro-deposición de oro. El estudio experimental, se desarrolló en tres etapas: Oxidación de Tiourea, Lixiviación de oro y Electro-deposición de oro. Como una alternativa a los agentes químicos, la oxidación de $T U$ se realizó vía electrólisis para producir Disulfuro Formamidina (FDS), el cual, sirve como oxidante en la disolución de oro. El grado de conversión óptima de tiourea a disulfuro formamidina se obtiene en 2,5 $\mathrm{h}$ aplicando una FEM de 4,5 V con un pH igual 1,5. Los compartimientos anódico y catódico del reactor electroquímico fueron separados por una membrana aniónica con la finalidad de eliminar reacciones indeseables en el cátodo. Previo a la etapa de lixiviación, el mineral fue lavado con ácido sulfúrico para eliminar los agentes consumidores de ácido y mantener de este modo un $\mathrm{pH}$ constante durante el tiempo que dure la lixiviación. Durante la lixiviación de oro, se estudió las velocidades de disolución en función de las concentraciones de TU, FDS y pH. Finalmente, se efectuó una prueba de lixiviación en tiempos prolongados usando los parámetros óptimos de operación obtenidos en las pruebas anteriores. La prueba de electro-deposición se llevó a cabo usando la técnica de electrodiálisis en una celda de tres compartimentos El bajo consumo de Tiourea (1,76 kg/TM mineral) demuestra que es una alternativa viable desde el punto de vista económico.
\end{abstract}

Palabras claves.- Electrolixiviación, Electrodeposición, Tiourea, Disulfuro formamidina.

\begin{abstract}
The research in the laboratory was made operating variables (reactant concentration, $p H$ and oxidation potential) of pyrite leaching gold from river Tablachaca (Ancash Peru) with thiourea (TU) followed by electro-deposition of gold. The experimental study was conducted in three stages: Oxidation of thiourea leaching of gold and gold electroplating. As an alternative to chemical agents, TU oxidation via electrolysis was conducted to obtain formamidine disulphide (FDS), which serves as an oxidizer in the dissolution of gold. The optimum degree of conversion of formamidine disulfide thiourea in $2.5 \mathrm{~h}$ was obtained by
\end{abstract}

\footnotetext{
${ }^{1}$ Egresado de la Facultad de Ingeniería Química y Textil de la Universidad Nacional de Ingeniería, ${ }^{2}$ Egresado de la Facultad de Ingeniería Química y Textil de la Universidad Nacional de Ingeniería, ${ }^{3}$ Ing. Docente de la Facultad de Ingeniería Química y Textil de la Universidad Nacional de Ingeniería.
} 
applying a FEM of $4.5 \mathrm{~V}$ with a $\mathrm{pH}$ of 1.5. The anodic and cathodic compartments of the electrochemical reactor were separated by an anion membrane with the purpose of eliminating undesired reactions at the cathode. Prior to the leaching step, the mineral was washed with sulfuric acid to remove the acid consuming agents and thereby maintain a constant pH throughout of the leach. During the leaching of gold, we studied the dissolution rates as a function of the concentrations of TU, SDS and $\mathrm{pH}$. Finally, a test was performed on prolonged leaching using the optimum operating parameters obtained in the previous tests. The electro-deposition test was carried out using the technique of electrodialysis in a threecompartment cell. The low consumption of thiourea (1.76 kg / MT mineral) proves to be a viable alternative economically

Keywords.- Electro leaching, Electroplating, Thiourea, Formamidine disulfide.

\section{INTRODUCCIÓN}

La TU tiene mayores ventajas que el cianuro sobre todo en minerales refractarios. Es el lixiviante alternativo que mayor atención ha recibido por parte de los investigadores interesados en la lixiviación de oro y plata ${ }^{[1,2,3]}$.Este interés se debe sobre todo a dos factores uno porque es mucho menos tóxica que el cianuro y dos por las altas tasas iníciales de disolución. Las principales características inherentes al proceso son: Operación en medio ácido( $\mathrm{pH} 1.5$ a 3.0), posibilidad de usar varios agentes oxidantes, entre ellos el $\mathrm{Fe}^{3+}$, la disolución del oro involucra la formación de un complejo catiónico a diferencia de la cianuración que es un complejo aniónico ${ }^{[2,3,4]}$ .La reacción global de disolución para la formación del complejo catiónico está representado por ${ }^{[5]}$ :

$$
A u(s)+2 C S(N H 2)_{2(a c)} \leftrightarrow\left[A u\left(C S\left(N_{2}\right)_{2}\right)_{2}\right]_{(a c)}^{+}+e^{-}, E^{\mathbf{o}}=0.38 V(S H E),
$$

La disolución del oro requiere un oxidante apropiado, tal como peróxido de hidrógeno, ión férrico, oxígeno o aire, dióxido de manganeso, etc.
Sin embargo, la Tiourea por sí misma es inestable bajo algunas condiciones oxidantes, y se descompone ${ }^{[3]}$.

$$
\begin{aligned}
& 2 \mathrm{CS}\left(\mathrm{NH}_{2}\right)_{2(a c)} \leftrightarrow\left(\mathrm{CSNH}_{2}(\mathrm{NH})\right)_{2(a c)}+2 \mathrm{H}^{+}(a c), E^{\mathrm{o}}=0.42 \mathrm{~V}(\mathrm{SHE}),(2) \\
& \left(\mathrm{CSNH}_{2}(\mathrm{NH})\right)_{2(a c)} \rightarrow \mathrm{CS}\left(\mathrm{NH}_{2}\right)_{2(a c)}+\text { componente } \quad \text { s.Sulf , (3) }
\end{aligned}
$$

La descomposición de la TU (2) es un proceso reversible y genera el Disulfuro Formamidina (FDS: $\left(\mathrm{NH}_{2}(\mathrm{NH}) \mathrm{CSSC}(\mathrm{NH}) \mathrm{NH}_{2}\right)$ ) . Bajo algunas condiciones, el (FDS) se descompone (3) relativamente lento a subproductos de (TU) y un componente sulfínico el cual puede rápidamente descomponerse en cianamida y azufre elemental. En soluciones ligeramente ácidas a neutras, la TU es relativamente estable $(\mathrm{pK}=4,3)$ pero en condiciones ácidas muy rigurosas se hidroliza para formar urea y gas sulfhídrico ${ }^{[4]}$. La lixiviación de minerales de oro con Tiourea normalmente es efectuada en valores de $\mathrm{pH}$ cercanos a $1,5^{[4]}$.En presencia de un oxidante, los productos de oxidación de Tiourea dependen del valor del $\mathrm{pH}$.A valores de $\mathrm{pH}$ por encima de 4,0, la Tiourea se oxida formando Disulfuro de Formamidina no protonado $\left(\mathrm{FDS} \mathrm{H}_{6}\right)$, extremadamente inestable (2). Pero en medio ácido $(\mathrm{pH}<4,0)$, la Tiourea se oxida formando Disulfuro de Formamidina protonado (FDS $\mathrm{H}_{8}{ }^{2+}$ ) o simplemente FDS $(4)^{[4]}$.

$$
2 \mathrm{Fe}^{3+}(a c)+2 \mathrm{CS}\left(\mathrm{NH}_{2}\right)_{2(a c)} \leftrightarrow\left[\mathrm{H}_{3} \mathrm{~N}(\mathrm{NH}) \mathrm{CSCS}(\mathrm{NH}) \mathrm{NH}_{3}\right]^{2+}{ }_{(a c)}+2 \mathrm{Fe}^{2+}{ }_{(a c)},(4)
$$

La semi-reacción para TU es (5):

$2 \mathrm{~S}\left(\mathrm{NH}_{2}\right)_{2}(a c) \leftrightarrow\left[\mathrm{H}_{3} \mathrm{~N}(\mathrm{NH}) \mathrm{CSSC}(\mathrm{HN}) \mathrm{NH}_{3}\right]^{2+}{ }_{(a c)}$
La formación de $\mathrm{FDSH}_{8}{ }^{2+}$ es un paso necesario para efectuar la lixiviación y por lo tanto no debe ser evitado; pero al ser menos estable que la Tiourea $\mathrm{y}$, en condiciones de oxidación muy severas, el FDS $\mathrm{H}_{8}{ }^{2+}$ podría oxidarse 
Determinación experimental de los parámetros óptimos de operación en el proceso de electrolixiviación y electrodeposición secuencial de oro en soluciones ácidas de tiourea a partir de sulfuros concentrados

irreversiblemente a urea y sulfato, haciendo que el proceso sea antieconómico. ${ }^{[4]}$ En medio alcalino, el $\mathrm{FDSH}_{6}$ se descompone para formar azufre elemental, TU y cianato(6).

$$
\left(\mathrm{SCNH}_{2}(\mathrm{NH})\right)_{2(u v)} \rightarrow \mathrm{CN}\left(\mathrm{NH}_{2}\right)_{(a c)}+\mathrm{S}_{(s]}^{0}+\mathrm{CS}(\mathrm{NH}
$$

La Tiourea forma complejos estables con ciertos iones de metales de transición. ${ }^{[6]}$, y la cinética y mecanismo de reacción de la lixiviación del oro, pueden explicarse teniendo en cuenta las siguientes ecuaciones químicas sobre un electrodo de carbón vitreo:

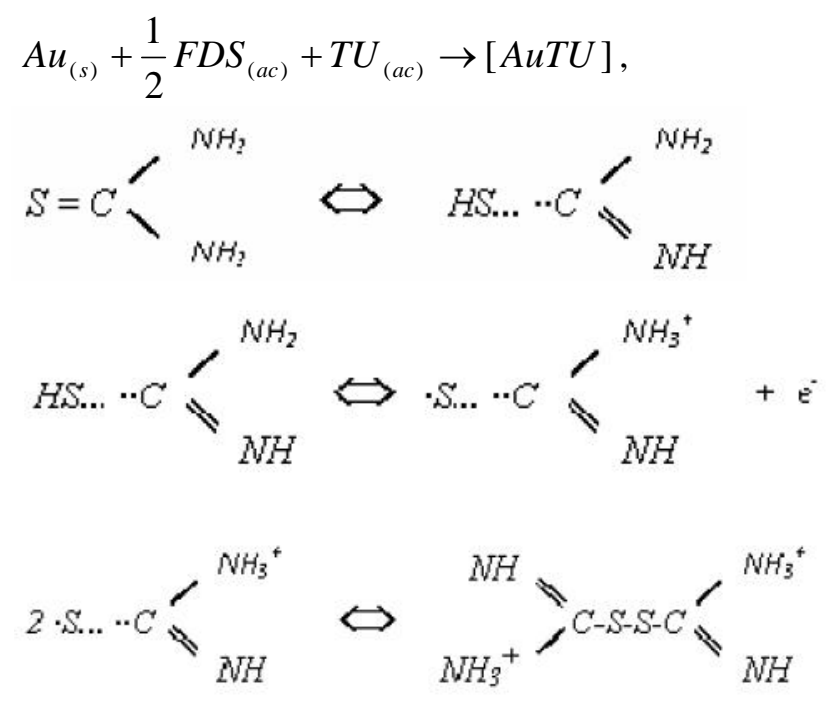

La ecuación (10), muestra como producto final el Disulfuro Formamidina protonado $\left(\mathrm{FDSH}_{8}{ }^{+}\right)$el cual conjuntamente con una molécula de Tiourea (TU) disuelven al oro tal como se muestra en la reacción (7).

La velocidad de reacción en el proceso de disolución de oro es de primer orden con respecto a la concentración de TU ${ }^{[3]}$. La cinética de reacción para la disolución de oro en una solución de TU ácida, está dada por (11):

$$
\frac{d C_{A u}}{d t}=k C_{T U} C_{F D S}^{0,5}
$$

La oxidación de TU empieza casi simultáneamente con la disolución de oro a $0,15 \mathrm{~V}$ respecto al electrodo de referencia de hidrógeno (SHE). La electro-disolución de oro en soluciones ácidas que contienen TU procede con $100 \%$ de eficiencia para $\mathrm{E}_{\text {ox }}^{0}<0,3 \mathrm{~V} / \mathrm{SHE}$, mientras que para $\mathrm{E}_{\mathrm{ox}}^{0}>0,3 \mathrm{~V}$, la reacción es acompañada por una electrooxidación de TU a FDS, azufre y trazas de sulfato ${ }^{[4,7]}$.
El incremento de temperatura en el proceso tiene doble afecto acelera la disolución de oro y degrada la TU lo que implica un mayor consumo ${ }^{[3,8]}$.

La temperatura determina que la energía de activación se sitúe dentro del rango en el cual el proceso es controlado por un fenómeno físico. $\mathrm{La}$ velocidad de reacción está limitada por un fenómeno de transporte cuando la velocidad de agitación está en un rango de [200 a 1200 rpm] ${ }^{[8] \text {. }}$

\section{Experimental}

Se describe los parámetros que afectan directamente en el proceso de tioureación, la metodología usada para estos análisis, los equipos y la instalación de los mismos. Cabe mencionar que por cuestiones prácticas y con la finalidad de simplificar la parte experimental, las pruebas de esta investigación están orientadas específicamente a determinar que parámetros son los que más influyen en el proceso de lixiviación con Tiourea. 
Como método de trabajo se uso la electrooxidación de la TU sobre carbón (usado en electroerosión) previa a la etapa de disolución de oro. El mineral estudiado es un concentrado sulfuro aurífero obtenido por concentración de arenas aluviales del río Tablachaca (Ancash Perú) en una mesa gravimétrica marca U-Tech modelo RP-4. Además de oro de granulometría relativamente gruesa (aproximadamente $2 \mathrm{~mm}$ ), el diseño de esta mesa permite concentrar metales valiosos de granulometría muy fina (alrededor de $1 \mathrm{~m}$ ) (Fig 1).

El peso total del concentrado fue $26 \mathrm{~kg}$ en todas las pruebas de lixiviación.

El análisis mineralógico se muestra en la Tabla1 y muestra los principales elementos que confieren refractariedad a un mineral. Los principales elementos que consumen una considerable cantidad de cianuro en el Proceso de Cianuración de minerales auríferos son azufre (S), arsénico $(\mathrm{As})$, cobre $(\mathrm{Cu})$ y en menor grado el hierro $(\mathrm{Fe})$.
El análisis granulométrico por lotes se muestra en la tabla 2.

La formación del Disulfuro Formamidina a partir de la oxidación de la TU fue obtenido por Oxidación electroquímica. De la curva corrientepotencial (Fig 2) se obtiene la FEM máxima para oxidar reversiblemete la TU. Para ello el primer paso fue obtener la curva voltamperométrica de oxidación. El segundo paso fue oxidar electroquímicamente diferentes cantidades de TU durante diversos periodos determinando la cantidad de FDS formado (Fig 3).

El tercer paso fue usar las soluciones tratadas para lixiviar el concentrado aurífero y evaluar las condiciones óptimas de operación que permita extraer más eficientemente el oro contenido en el mineral.

El último paso fue efectuar la electrodeposición [9,10,11] considerando un consumo mínimo de reactivos.

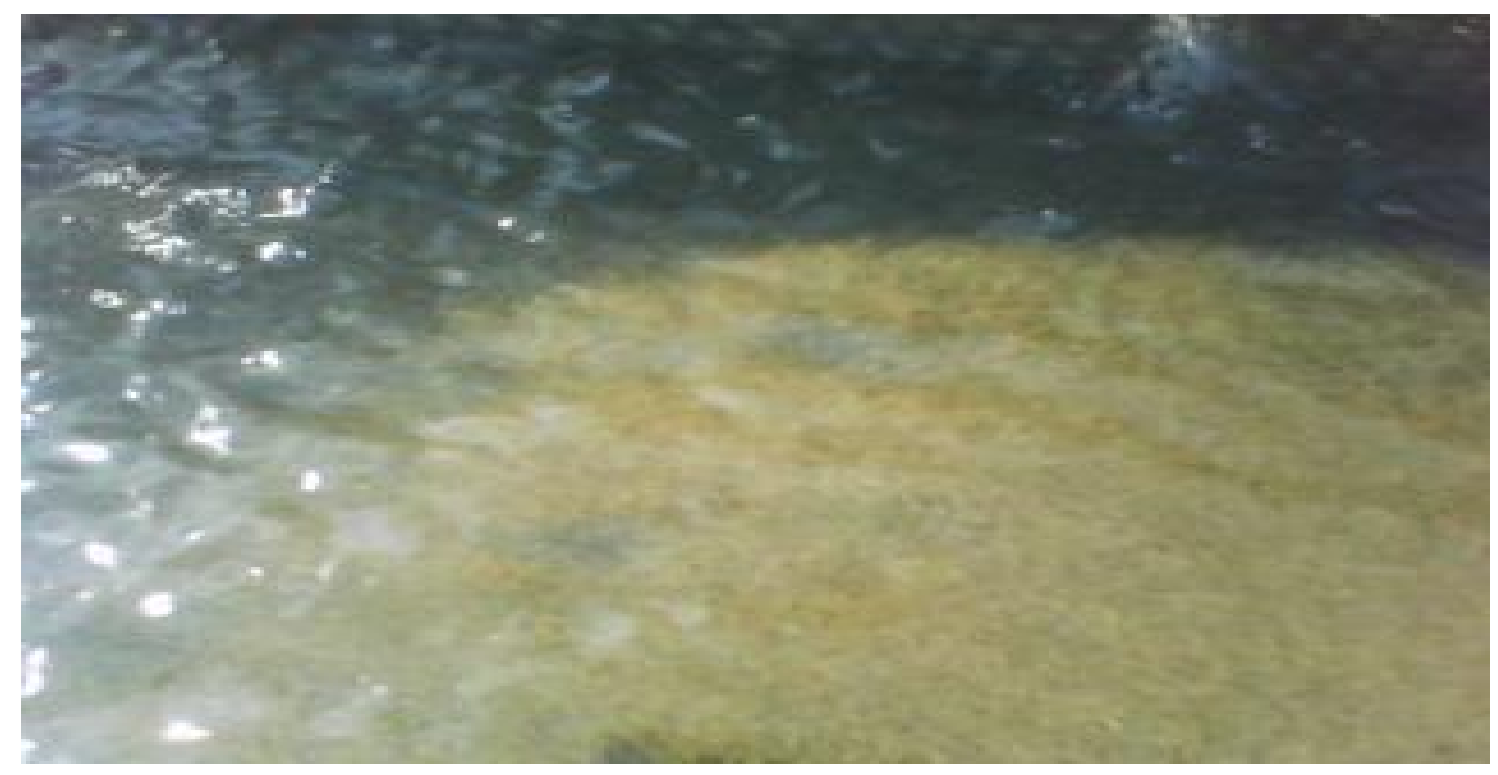

Fig. 1 Concentrado aurífero sobre la superficie de la mesa gravimétrica U-Techl RP-4.

Tabla 1. Composición mineralógica del concentrado aurífero ( $0.5 \mathrm{~kg}$ concentrado), río Tablachaca (Ancash Perú). 
Determinación experimental de los parámetros óptimos de operación en el proceso de electrolixiviación y electrodeposición secuencial de oro en soluciones ácidas de tiourea a partir de sulfuros concentrados

\begin{tabular}{lll}
\hline & Elemento & \multicolumn{1}{c}{ Concentración } \\
\hline & Azufre total & $18,91 \%$ \\
(S) & & \\
& Arsénico & $4,22 \%$ \\
(As) & & \\
& Antimonio & $0,23 \%$ \\
(Sb) & & $0,85 \%$ \\
& Cobre $(\mathrm{Cu})$ & $28,54 \%$ \\
& Hierro $(\mathrm{Fe})$ & $337,24 \mathrm{~g} / \mathrm{TM}$ \\
& Oro $(\mathrm{Au})$ & $52,36 \mathrm{~g} / \mathrm{TM}$ \\
& Plata $(\mathrm{Ag})$ & \\
\hline
\end{tabular}

Tabla 2. Granulometría del concentrado molido durante 45 min. Río Tablachaca, (Ancash Perú).

\begin{tabular}{lllr}
\hline & \multicolumn{2}{c}{ Malla } & \% Retenido \\
& + & 120 & 12,40 \\
Mesh & & & \\
& + & 140 & 26,60 \\
Mesh & & & \\
Mesh & - & 140 & 61,00 \\
\hline
\end{tabular}

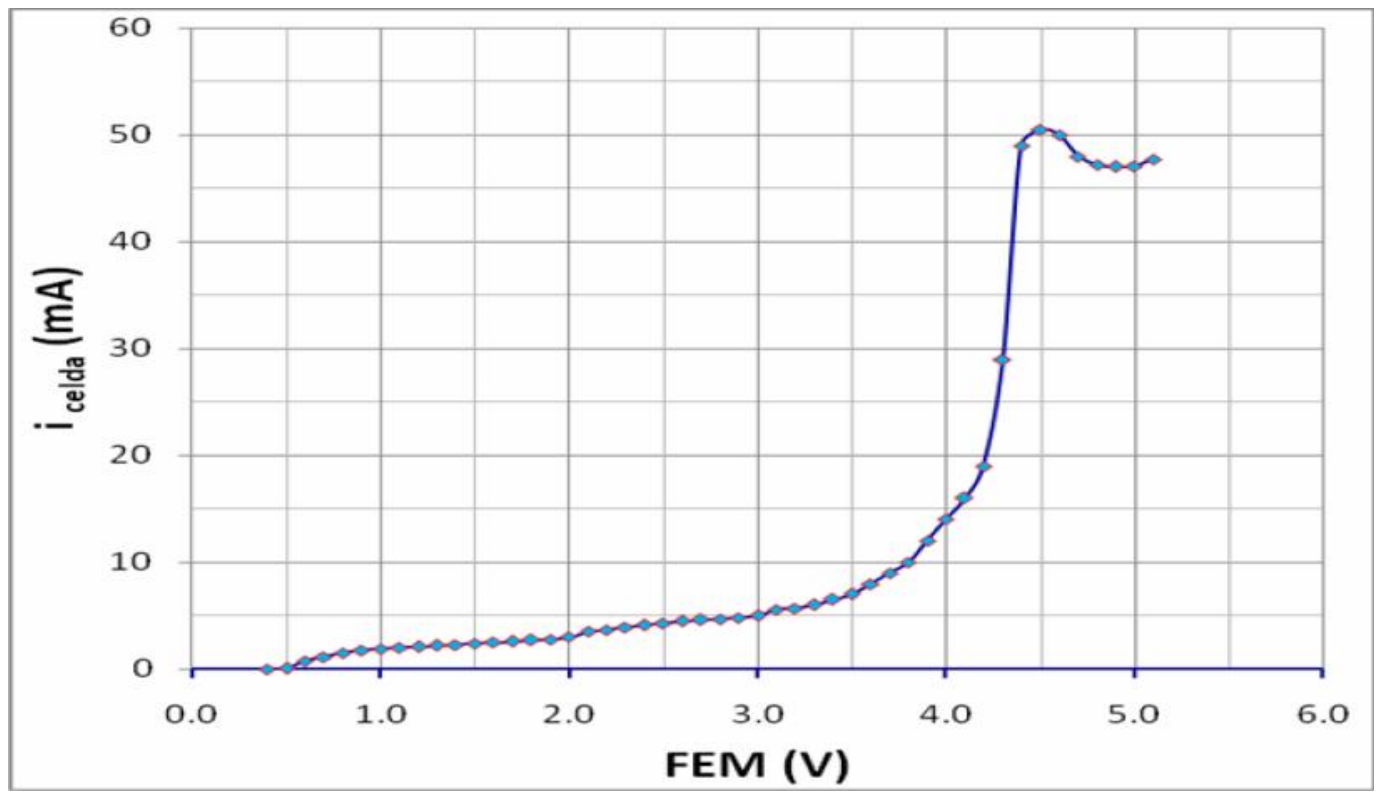

Fig. 2 Corriente Anódica en función de FEM aplicada. Electrodo de Ag/AgCl, 3.5M KCl. 


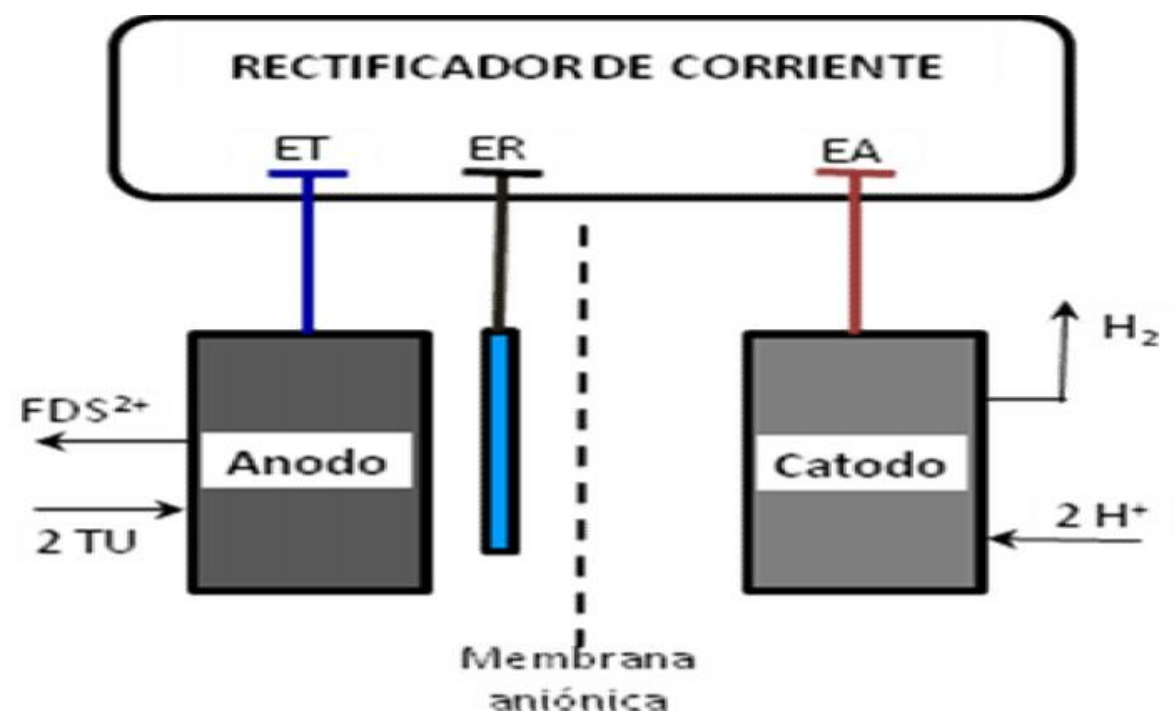

Fig. 3 Esquema del reactor de oxidación de TU a FDS. ET: electrodo de trabajo (ánodo), ER: electrodo de referencia ( $\mathrm{Ag} / \mathrm{AgCl} \mathrm{KCl}$ 3,5 M), EA: electrodo auxiliar (cátodo).

Los datos experimentales obtenidos en la prueba de electro-oxidación de TU se muestra en la Fig 4 a $\mathrm{pH}=1.52$, FEM=4.5 (Fig 2). La diferencia entre los valores de TU total y TU libre representa la concentración de FDS formado. Para determinar si el grado de conversión depende de la concentración inicial de la sustancia reactante, se realizaron tres pruebas de electro-oxidación durante $2 \mathrm{~h}$ con diferentes cantidades de TU, los resultados se muestran en la tabla 3 .

Al final de cada experimento se tomó una muestra de cada prueba para determinar la concentración de TU libre y $\mathrm{TU}$ total que permiten la determinación del grado de conversión de cada prueba.

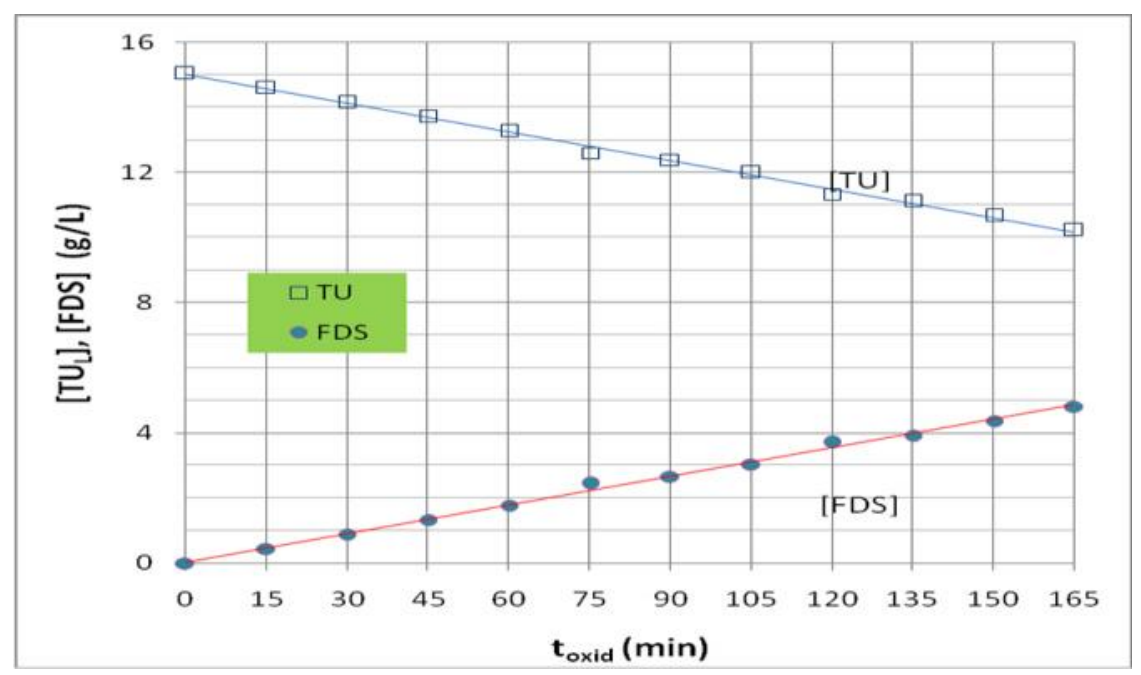

Fig. 4 Electro- oxidación de TU a FDS. $P M_{T U}=76,12 \mathrm{~g} / \mathrm{mol}, P M_{K I O 3}=214 \mathrm{~g} / \mathrm{mol}, P M_{F D S}=152,24 \mathrm{~g} / \mathrm{mol}$. 
Determinación experimental de los parámetros óptimos de operación en el proceso de electrolixiviación y electrodeposición secuencial de oro en soluciones ácidas de tiourea a partir de sulfuros concentrados

Tabla 3. Conversión en función del peso inicial de TU.

\begin{tabular}{ccrrrrrrr}
\hline Prueba & $\mathbf{W}_{\text {TU bruto }}(\mathbf{g})$ & \multirow{2}{*}{$\mathbf{W}_{\text {TU neto }}(\mathbf{g})$} & Vsol $(\mathbf{m L})$ & {$\left[\mathbf{T U}_{\mathbf{T}}\right]_{\mathbf{i}}(\mathbf{g} / \mathbf{L})$} & $\begin{array}{c}\text { Tiempo } \\
\text { Oxid(min) }\end{array}$ & $\mathbf{T U}_{\mathbf{L}}$ & $\mathbf{T U}_{\mathbf{T}}$ \\
\hline 1 & 20,1385 & 20,0056 & 2000 & 10,0028 & 120 & 0,34 & 0,90 \\
2 & 45,4216 & 45,1218 & 3000 & 15,0406 & 120 & 0,51 & 1,35 \\
3 & 60,4005 & 60,0019 & 3000 & 20,0006 & 120 & 0,68 & 1,80 \\
\hline
\end{tabular}

La lixiviación del concentrado aurífero se realizó usando una relación sólido/líquido para todas la pruebas de 1:2. Para determinar la influencia de la concentración inicial de TU libre sobre la disolución de oro, se efectuaron tres pruebas de lixiviación con soluciones de similar concentración de FDS, obtenidas por electro-oxidación de cantidades diferentes de TU disuelta.

Las concentraciones de oro fueron obtenidas por absorción atómica (Equipo Shimadzu AA-6701F). Los datos experimentales se muestran en la Fig 5.

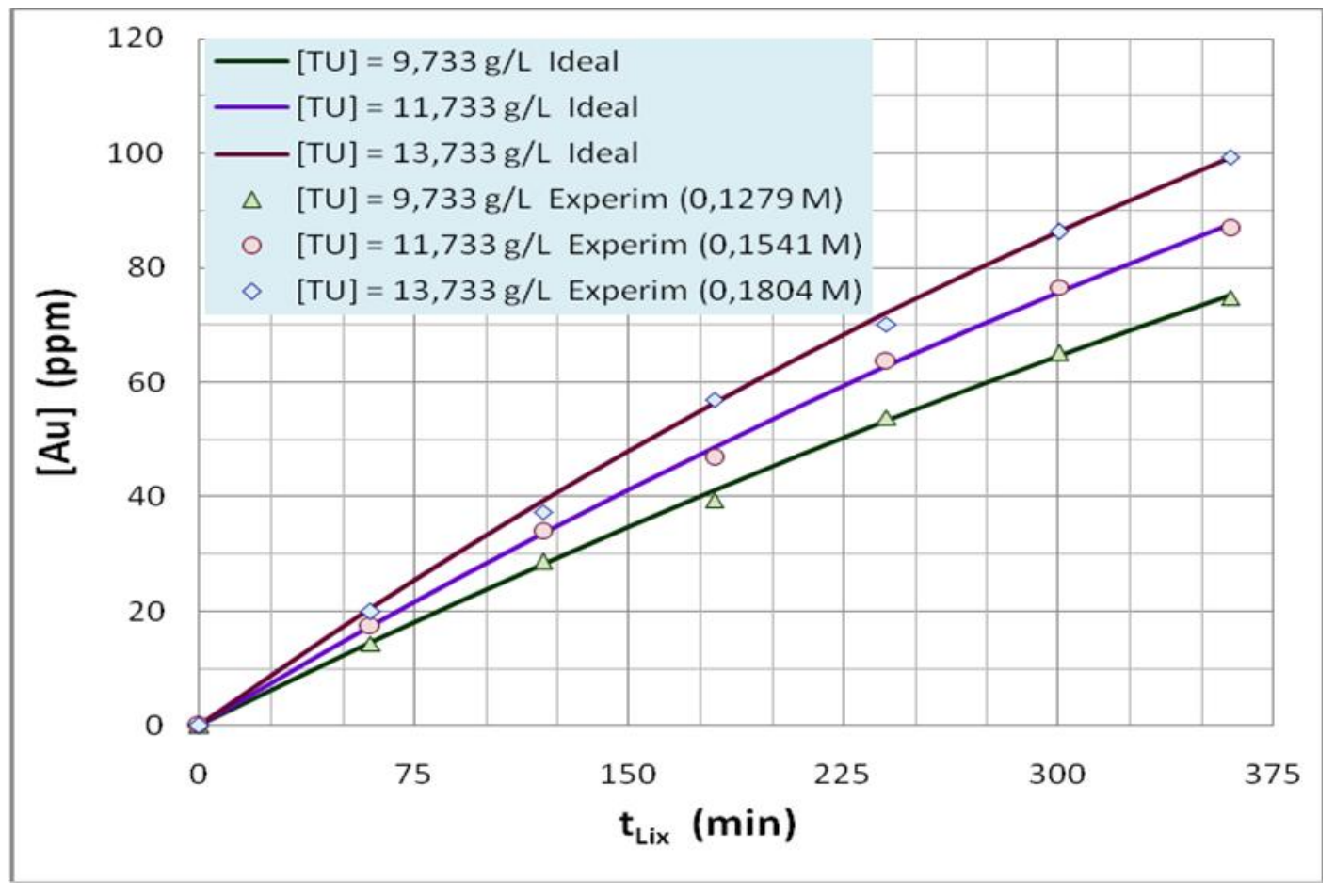

Fig. 5 Concentración de Au en solución con FDS constante. $[F D S]_{0}=3,268 \mathrm{~g} / \mathrm{L}(0,0215 \mathrm{M}), \mathrm{pH}=1,51 . p H=$ 1,51, Peso mineral $=1.5 \mathrm{~kg}$, Peso oro $=0.50586 \mathrm{~g}, \mathrm{Vsol}=3004 \mathrm{~mL}$.

La influencia de la concentración inicial de Disulfuro Formamidina, fue estudiada a partir de 3 soluciones preparadas con diferentes cantidades de TU de modo tal que al final de la electrooxidación se obtuvieron similares concentraciones de TU libre y diferentes concentraciones de FDS. La Fig. 6 muestra los resultados. En la Fig 7 se muestra la tendencia lineal de las concentraciones de Au en función de las concentraciones iniciales de TU libre al cabo de 1 h de lixiviación.

La Fig 8 muestra la curva de lixiviación de oro con parámetros óptimos de operación. La cinética y orden de reacción fue estudiada, sin embargo no forma parte de este artículo, será presentado en otro artículo. 


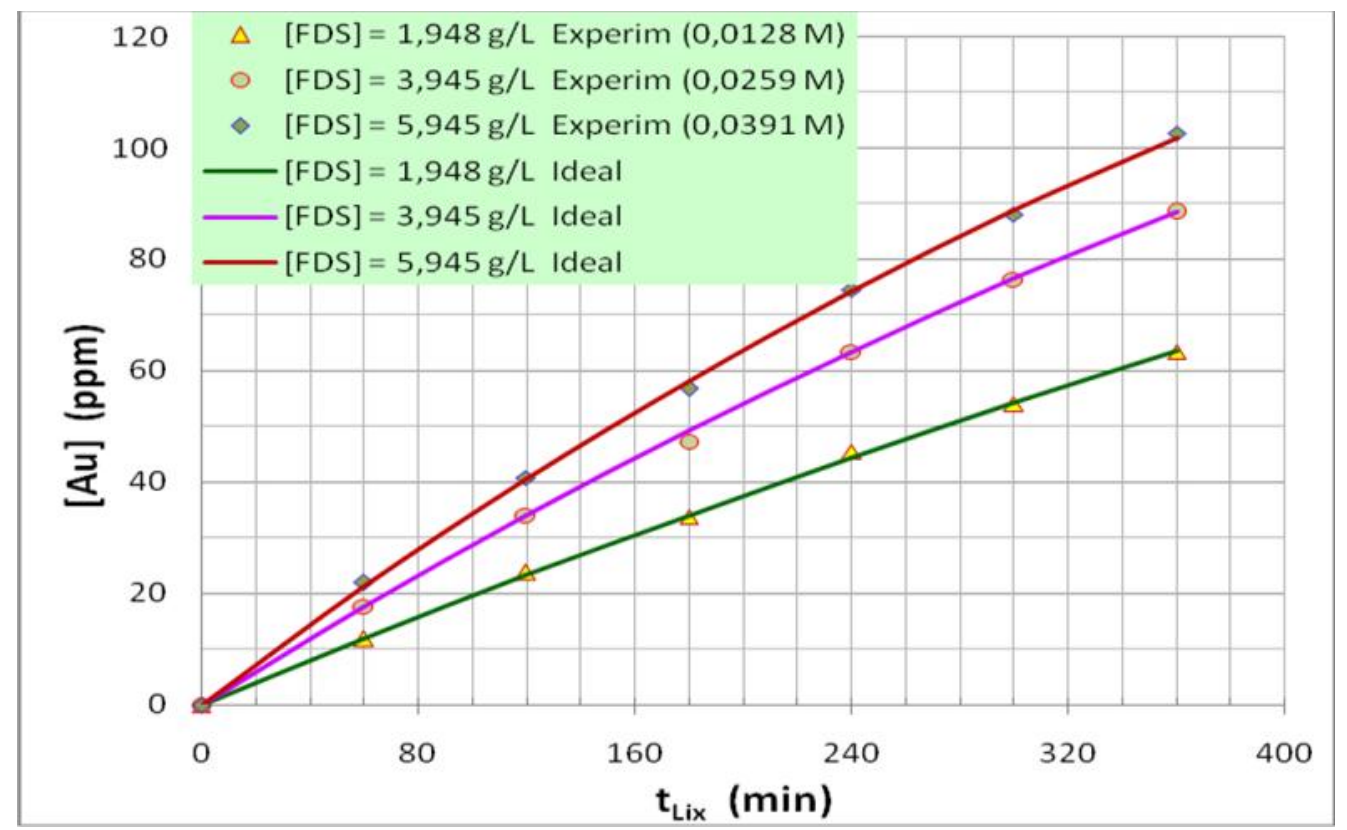

Fig. 6 Extracción de Au con TU constante. $\left[T U_{L}\right]_{0}=11,055 \mathrm{~g} / \mathrm{L}(0,1452 \mathrm{M}), \mathrm{pH}=1,52$, Peso mineral $=1,5$ $\mathrm{kg}$, Peso de oro $=0,50586 \mathrm{~g}$.

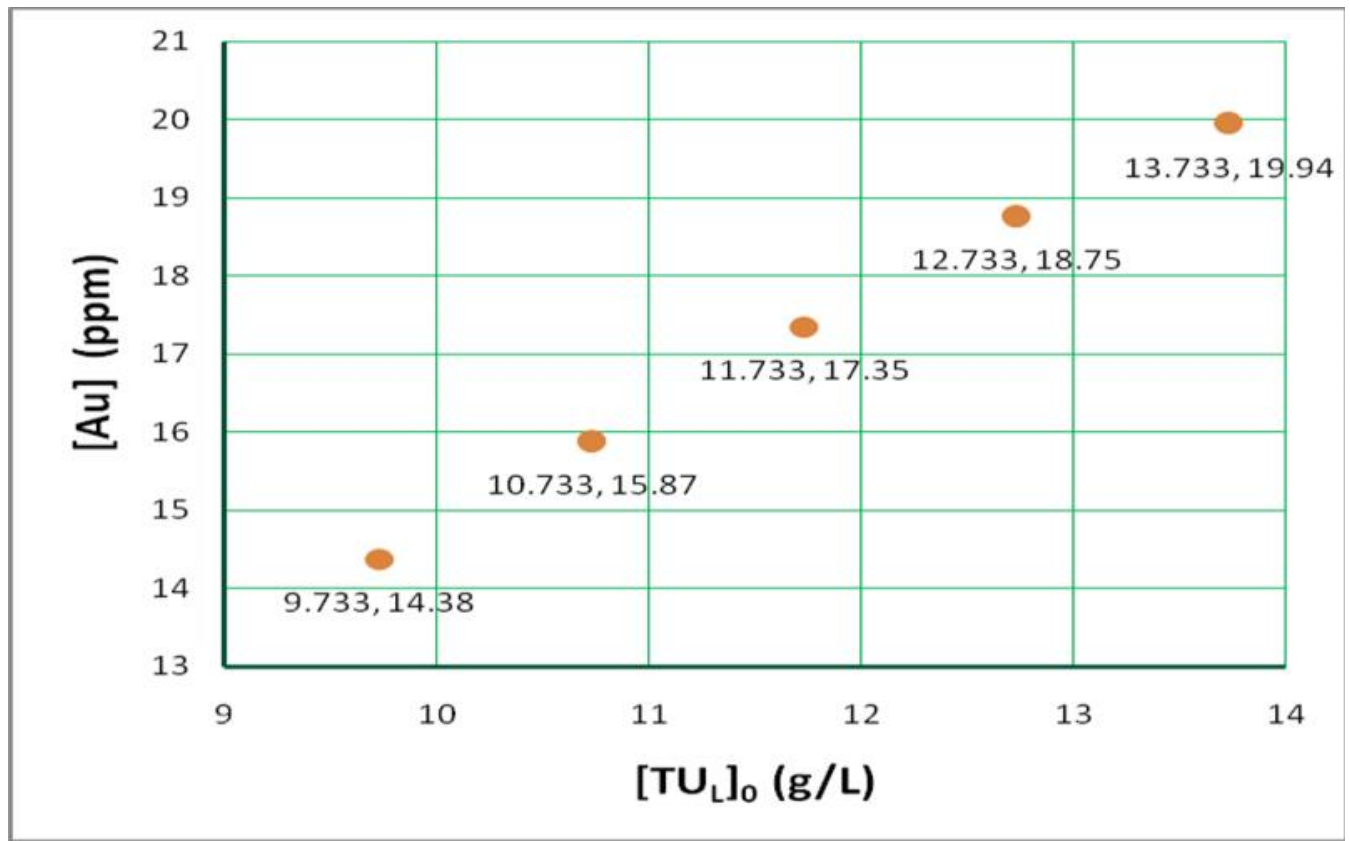

Fig 7. Concentración de Au en una hora de lixiviación para diferentes concentraciones iniciales de TU libre. 
Determinación experimental de los parámetros óptimos de operación en el proceso de electrolixiviación y electrodeposición secuencial de oro en soluciones ácidas de tiourea a partir de sulfuros concentrados

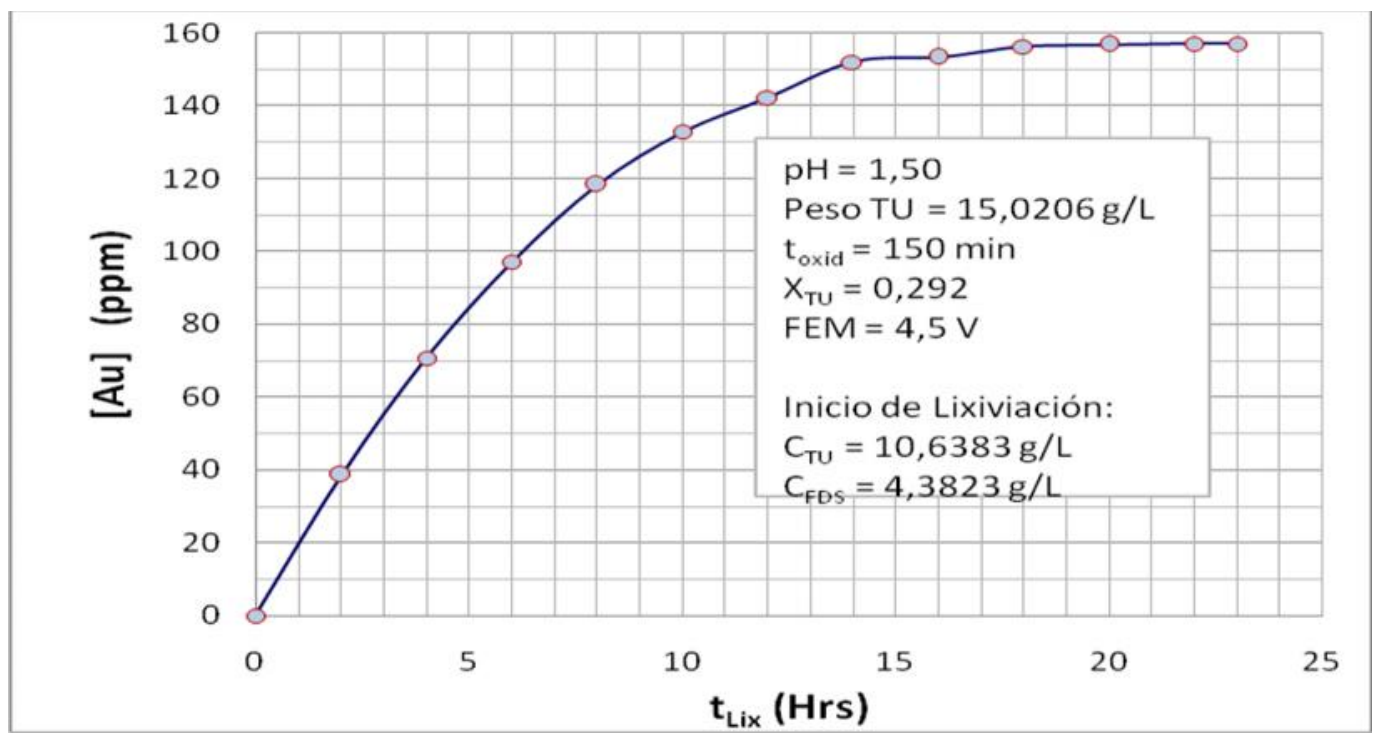

Fig. 9 Concentración de oro (ppm) en lixiviación por tiempos prolongados (hrs).

\section{RESULTADOS Y DISCUSIÓN}

En esta investigación se determinó que, de los varios mecanismos de reacción de electrodo propuestos, los que mejor satisfacen los resultados experimentales son aquellos que involucran la adsorción de especies electro-activas y un establecimiento lento del equilibrio de adsorción antes de la reacción electroquímica. Las especies adsorbidas son reducidas más fácilmente que las especies en solución, esto quiere decir que la reducción del complejo $\left[\mathrm{Au}(\mathrm{TU})_{2}\right]^{+}$se lleva a cabo en dos etapas, proceso que puede ser explicado mediante las siguientes ecuaciones.

$$
\begin{gathered}
{\left[A u(T U)_{2}\right]^{+} \leftrightarrow\left[A u(T U)_{2}\right]_{a d}^{+}} \\
\text {químicas. }\left[A u(T U)_{2}\right]_{a d}^{+}+e^{-} \rightarrow A u_{(s)}+2 T U \\
{\left[A u(T U)_{2}\right]^{+}+e_{s o l}+e^{-} \rightarrow A u(S)+2 T U}
\end{gathered}
$$

Los ensayos de lixiviación muestran a mayor concentración de TU, mayor es la velocidad de lixiviación inicial de oro. Este comportamiento no es igual respecto al FDS, pues; solo cuando la concentración es mayor o igual a $4 \mathrm{~g} / \mathrm{L}$ aumenta apreciablemente la velocidad de disolución. Se encontró que la electro-disolución de oro en soluciones ácidas que contienen TU procede con $100 \%$ de eficiencia para $\mathrm{E}_{\text {ox }}^{0}<0,3 \mathrm{~V}$ (SHE), mientras que para $\mathrm{E}_{\text {ox }}^{0}>0,3 \mathrm{~V}$, la reacción es acompañada por una electro-oxidación de TU a FDS, azufre y trazas de sulfato. La eficiencia de corriente en las reacciones de electrodeposición disminuye a medida que la concentración de iones metálicos disminuye, por ello es aconsejable llevar a cabo la operación con soluciones cuya concentración de oro sea por lo menos 10 ppm o $5,08 \times 10^{-5} \mathrm{M}$ como $\left[\mathrm{AuTU}_{2}\right]^{+}$. Dado que en la mayoría de los casos, la lixiviación se lleva a cabo con minerales auríferos de baja ley, entonces se obtendrá soluciones impregnadas de bajo valor en oro. Por lo tanto, para elevar la concentración de oro en solución es necesario proceder previamente con una adsorción de la solución impregnada ya sea con carbón activado o con resinas de intercambio iónico y posteriormente someter a una elución para obtener una solución enriquecida con alto contenido aurífero que permita mantener una eficiencia de corriente aceptable durante la electrodeposición de oro.

\section{CONCLUSIÓN}

Los parámetros óptimos para el proceso de lixiviación del oro en soluciones ácidas de Tiourea 
determinados experimentalmente son: $\mathrm{C}_{\mathrm{Au}} 10 \mathrm{ppm}$, $\mathrm{C}_{\mathrm{TU}} 10,6 \mathrm{~g} / \mathrm{L}, \mathrm{C}_{\mathrm{FDS}} 4,4 \mathrm{~g} / \mathrm{L}, \mathrm{pH} 1,5$, FEM 4,5.

\section{AGRADECIMIENTO}

A la Universidad Nacional de Ingeniería Facultad de Ingeniería Química y Textil (UNIFIQT) por el apoyo brindado, en especial al jefe del Laboratorio de Fisicoquímica N ${ }^{\circ} 11$ Prof. W. Reátegui R. por su apoyo como asesor de tesis, así como durante el desarrollo experimental.

\section{REFERENCIAS}

1. Gos, S., Rubo, A., "The Relevance of Alternative Lixiviants With Regard to Technical Aspects", Work Safety and Environmental Safety. Degussa AG, Rodenbacher Chaussee 4, Hannau, Germany.

2. Deschenes, G., "Investigation on the Potential Techniques to Recover Gold From Thiourea Solution". Gold Metallurgy, Mineral Sciences Laboratories. CANMET, Energy, Mines and Resources Canada.

3. Li, J., Miller, J. D., "Reaction Kinetics for Gold Dissolution in Acid Thiourea Solution Using Formamidine Disulfide as Oxidant". SME Annual Meeting. Preprint 99-62. 1999, Denver, Colorado.

4. Roberto De B. Emery Trindate. "Tiouréia e Bromo como Lixiviantes Alternativos à Cianetação do Ouro". Série Tecnología Mineral, Rio de Janeiro, n. 67, 1994.

5. Aguayo Salinas, S., Encinas Romero, M. A., GONZALES. I., "Electrochemical
Dissolution of Calaverite $\left(\mathrm{AuTe}_{2}\right)$ in Thiourea Acidic Solutions". 1996. Journal of Applied Electrochemistry 28 (1998) 417-422.

6. Harvanová J., "The Electrochemical Study of Thiourea Behaviour by Cyclic Voltamperometry". Acta Metallurgica Slovaca, 4, Special Issue 4/2001, (58 - 61).

7. Bolzán, A. E., Iwasita, T. Arvia. A. J. "In situ FTIRRAS study of the electro-oxidation reactions of thiourea and gold in aqueous acid solutions". Journal of Electroanayitical Chemistry 554-555 (2001) 49-60. Science Direct.

8. Misari, F. S., Franco Jiménez, J. A., "Metalurgia del Oro". V-II, Centro de Estudios y Promoción en Ciencias de la Tierra, 1993.

9. Zhang, H., Ritchie, I. M., La Brooy, S. R., "Electrochemical Oxidation of Gold and Thiourea in Acidic Thiourea Solutions". Journal of The Electrochemical Society. 148 (10) D146-D153 (2001).

10. Bolzán, A. E., Iwasita, T., Arvia A. J., "In situ FTIRRAS study of the electro-oxidation reactions of thiourea and gold in aqueous acid solutions". Journal of Electroanayitical Chemistry 554-555 (2001) 49-60. Science Direct.

11. Juarez, M., Dutra. A. J. B., "Gold Electrowinning from Thiourea Solutions. Minerals Engineering", Elsevier Science Ltd.Vol. 13, No. 10-11, pp. 1083-1096, 2000.

Correspondencia: wreategui@uni.edu.pe 